\title{
PIECEWISE LINEAR DIFFERENTIAL SYSTEMS WITHOUT EQUILIBRIA PRODUCE LIMIT CYCLES?
}

\author{
JAUME LLIBRE ${ }^{1}$ AND MARCO ANTONIO TEIXEIRA ${ }^{2}$
}

\begin{abstract}
In this article we study the planar piecewise differential systems formed by two linear differential systems separated by a straight line, such that both linear differential have no equilibria, neither real nor virtual.

When the piecewise differential system is continuous, we show that the system has no limit cycles. But when the piecewise differential system is discontinuous, we show that it can have at most one limit cycle.
\end{abstract}

\section{Introduction AND STATEMENT OF the MAIN RESUlt}

The study of the so-called discontinuous linear differential systems goes back to Andronov, Vitt and Khaikin [1] and have received special attention from the mathematical community nowadays, mainly because these systems are widely used to model processes appearing in electronics, mechanics, economy, etc. See, for instance, the books of di Bernardo, Budd, Champneys and Kowalczyk [5] and Simpson [28], the survey of Makarenkov and Lamb [26], and the hundreds of references which appear in these last three cited works.

The simplest possible piecewise linear differential systems are the ones formed by two linear differential systems separated by a straight line. We note that for these apparently simple systems, when they are continuous, some serious work is necessary for proving that they have at most one limit cycle, see [7] and [21]. This solved the conjecture of Lum and Chua [25] done in 1990 that such continuous differential systems can have at most one limit cycle.

The study of the maximum number of limit cycles of these systems, when they are discontinuous, still is an open question. Up to now we know that there are discontinuous systems with at least three limit cycles, see for instance $[2,4,3,6,8,9,10,11,12,13,14,15,22,17,18,19,20,22,24]$.

We consider planar piecewise differential systems formed by two linear differential systems separated by the straight line $x=0$, such that both linear differential have no equilibria, neither real nor virtual. We say that

2010 Mathematics Subject Classification. 34C29, 34C25, 47H11.

Key words and phrases. limit cycles, continuous piecewise linear differential systems, discontinuous piecewise differential systems. 
an equilibrium point $\left(x_{0}, y_{0}\right)$ of the linear differential system defined in the half-plane $x \geq 0$ is real if it $x_{0} \geq 0$, otherwise it is called virtual.

Theorem 1. Continuous planar piecewise differential systems formed by two linear differential systems separated by a straight line, such that both linear differential have no equilibria, neither real nor virtual, have no limit cycles.

Theorem 1 is proved in section 2 .

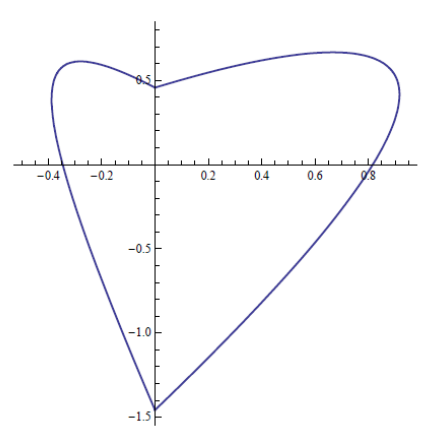

FiguRE 1. The limit cycle of the piecewise linear differential system stated in Theorem 2.

Theorem 2. Discontinuous planar piecewise differential systems formed by two linear differential systems separated by a straight line, such that both linear differential have no equilibria, neither real nor virtual, have at most one limit cycle, and the next example shows that there are piecewise linear differential systems with one limit cycle: The piecewise linear differential system

$$
\begin{array}{ll}
\dot{x}=x-y-\frac{1}{2}, \quad \text { if } x>0, \text { and } \quad & \dot{x}=-x-y, \\
\dot{y}=x-y, & \dot{y}=3 x+3 y-1, \quad \text { if } x<0
\end{array}
$$

has the limit cycle of Figure 2.

Theorem 2 is proved in section 3 .

Remark 3. Concerning Theorem 2, we stress that a closed curve, consisting of union of branches of trajectories of both systems, one in $x>0$ and the other in $x<0$, passing through points of the closure of the sliding region is not considered a limit cycle. For a definition of sliding region see for instance [5]. 


\section{Continuous piecewise differential system: Proof of THEOREM 1}

We consider in the half-plane $x>0$ the following general linear differential system without equilibria

$$
\begin{aligned}
& \dot{x}=\alpha x+\beta y+\gamma=P_{+}(x, y), \\
& \dot{y}=\lambda \alpha x+\lambda \beta y+\delta=Q_{+}(x, y),
\end{aligned}
$$

and in the half-plane $x<0$ another general linear differential system without equilibria

$$
\begin{aligned}
& \dot{x}=a x+b y+c=P_{-}(x, y), \\
& \dot{y}=\mu a x+\mu b y+d=Q_{-}(x, y) .
\end{aligned}
$$

Here the dot denotes derivative with respect to the independent variable $t$ usually called the time. Changing the sign of the time (if necessary) we can assume without loss of generality that

$$
\lambda \beta \geq 0 .
$$

In order that both linear differential systems define in the whole plane $\mathbb{R}^{2}$ a continuous differential system we must take

$$
\beta=b, \quad \gamma=c, \quad \mu=\lambda, \quad \delta=d .
$$

We denote the divergence of system $(j)$ by $\operatorname{Div}_{j}$, thus we have

$$
\operatorname{Div}_{1}=\alpha+\lambda b, \quad \operatorname{Div}_{2}=a+\lambda b .
$$

We note that in order that the continuous piecewise linear differential systems in $\mathbb{R}^{2}$ formed by systems (1) and (2) can have periodic orbits it is necessary that either $\operatorname{Div}_{1} \operatorname{Div}_{2}<0$, or $\operatorname{Div}_{1}=\operatorname{Div}_{2}=0$. This follows from the following claim: If either $\operatorname{Div}_{1} \operatorname{Div}_{2}>0$, or $\operatorname{Div}_{i} \neq 0$ and $\operatorname{Div}_{j}=0$ for $i \neq j$, then the continuous piecewise linear differential systems in $\mathbb{R}^{2}$ formed by systems (1) and (2) have no periodic solutions. Now we prove the claim.

Clearly if there is a limit cycle this must cross the straight line of separation $x=0$ between the two linear differential system because linear differential systems have no limit cycles. The vector field associated to our differential system is

$$
X(x, y)= \begin{cases}\left(P_{+}(x, y), Q_{+}(x, y)\right) & \text { if } x>0, \\ \left(P_{-}(x, y), Q_{-}(x, y)\right) & \text { if } x<0 .\end{cases}
$$

We shall use the following well-known result, the Green's theorem, for a proof see for instance [27].

Theorem 4. Let $\gamma$ be a piecewise smooth, simple closed curve in $\mathbb{R}^{2}$, and let $R$ be the open region bounded by $\gamma$. If $P=P(x, y)$ and $Q=Q(x, y)$ are functions defined on an open region containing $R$ and have continuous partial derivatives there, then

$$
\oint_{\gamma}(P d y-Q d x)=\iint_{R}\left(\frac{\partial P}{\partial x}+\frac{\partial Q}{\partial y}\right) d x d y
$$


where the integration path along $\gamma$ is in counterclockwise sense.

The divergence of a $\mathcal{C}^{1}$ differential system

$$
\dot{x}=P(x, y), \quad \dot{y}=Q(x, y),
$$

is the function

$$
\operatorname{div}(x, y)=\frac{\partial P}{\partial x}+\frac{\partial Q}{\partial y} .
$$

Let $\gamma$ be a periodic solution of the vector field $X(x, y)$, and let $R$ be the bounded region limited by $\gamma$. In order to apply the Green's theorem to this curve $\gamma$ and to the region $R$, we shall split such an integral as limit of two integrals as follows. We add to the periodic orbit $\gamma$ the segment $S$ of the $y$-axis contained in the region bounded by $\gamma$, now we split this segment as limit of two parallel segments $S_{-}(\varepsilon)$ and $S_{+}(\varepsilon)$ contained in $x<0$ and $x>0$ and at a distance $\varepsilon>0$ of $S$, respectively, and such that a piece $\gamma_{-}(\varepsilon)$ of $\gamma$ contained in $x<0$ together with $S_{-}(\varepsilon)$ forms an oval $O_{-}(\varepsilon)$. Similarly, we consider a piece $\gamma_{+}(\varepsilon)$ of $\gamma$ contained in $x>0$ such that together with $S_{+}(\varepsilon)$ forms another oval $O_{+}(\varepsilon)$, in such a way that the union of these ovals tends to $\gamma \cup S$ when $\varepsilon \mapsto 0$.

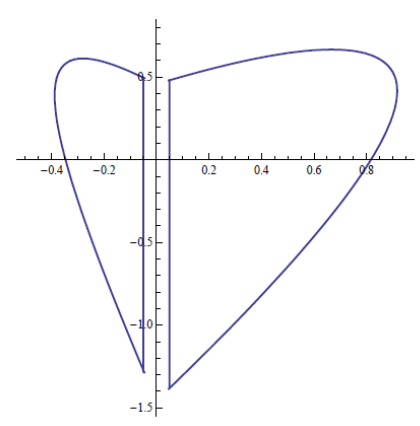

FiguRE 2. The ovals $O_{-}(\varepsilon)$ in $x<0$, and $O_{+}(\varepsilon)$ in $x>0$.

Clearly the two integrals

$$
\oint_{O_{-}(\varepsilon)}(P d y-Q d x) \text { and } \oint_{O_{+}(\varepsilon)}(P d y-Q d x)
$$

are well defined, and the integral

$$
\oint_{\gamma}(P d y-Q d x)
$$

is the limit when $\varepsilon \mapsto 0$ of

$$
I_{\varepsilon}=\oint_{O_{-}(\varepsilon)}(P d y-Q d x)+\oint_{O_{+}(\varepsilon)}(P d y-Q d x) .
$$


Applying the Green's theorem (Theorem 4) to both integrals of (5) we obtain that

$$
I_{\varepsilon}=\oint_{R_{-}(\varepsilon)}\left(\frac{\partial P}{\partial x}+\frac{\partial Q}{\partial y}\right) d x d y+\oint_{R_{+}(\varepsilon)}\left(\frac{\partial P}{\partial x}+\frac{\partial Q}{\partial y}\right) d x d y
$$

where $R_{ \pm(\varepsilon)}$ are the open regions bounded by the ovals $O_{ \pm(\varepsilon)}$. Now, from (5) and (6) the limit of $I_{\varepsilon}$ when $\varepsilon \mapsto 0$ is

$$
\oint_{\gamma}(P d y-Q d x)=\iint_{R}\left(\frac{\partial P}{\partial x}+\frac{\partial Q}{\partial y}\right) d x d y
$$

Since $\gamma$ is a periodic solution of the vector field $X(x, y)$ we have that $P d y-$ $Q d x=0$, so

$$
\oint_{\gamma}(P d y-Q d x)=0
$$

Consequently, if $R_{-}=R \cap\{x<0\}$ and $R_{+}=R \cap\{x>0\}$, then

$$
\iint_{R}\left(\frac{\partial P}{\partial x}+\frac{\partial Q}{\partial y}\right) d x d y=\iint_{R_{+}} \operatorname{Div}_{1} d x d y+\iint_{R_{-}} \operatorname{Div}_{2} d x d y=0,
$$

which is a contradiction with the assumptions that either $\operatorname{Div}_{1} \operatorname{Div}_{2}>0$, or $\operatorname{Div}_{i} \neq 0$ and $\operatorname{Div}_{j}=0$ for $i \neq j$. Hence the claim is proved.

Now in order to prove Theorem 1 it remains to show that when either $\operatorname{Div}_{1}=\operatorname{Div}_{2}=0$, or $\operatorname{Div}_{1} \operatorname{Div}_{2}<0$ the vector field $X(x, y)$ has no periodic solution, we distinguish two cases.

Case 1: $\operatorname{Div}_{1}=\operatorname{Div}_{2}=0$. Then, the piecewise differential system (1) and (2) becomes the linear differential system

$$
\begin{aligned}
& \dot{x}=-\lambda b x+b y+c, \\
& \dot{y}=-\lambda^{2} b x+\lambda b y+d,
\end{aligned}
$$

which clearly has no periodic solutions.

Case 2: $\operatorname{Div}_{1} \operatorname{Div}_{2}<0$. Without loss of generality we can assume that $\operatorname{Div}_{1}=\alpha+b \lambda>0$ and $\operatorname{Div}_{2}=a+b \lambda<0$, otherwise we reverse the sign of the independent variable in the differential system. Then, the solution $\left(x_{+}(t), y_{+}(t)\right)$ of system $(1)$ with the initial condition $\left(x_{+}(0), y_{+}(0)\right)=\left(0, y_{0}\right)$ is

$$
\begin{aligned}
x_{+}(t)= & \frac{1}{(\alpha+b \lambda)^{2}}\left(-c \alpha-b\left((\alpha+b \lambda)\left(y_{0}-c t \lambda\right)+d(t(\alpha+b \lambda)+1)\right)\right. \\
& \left.+e^{t(\alpha+b \lambda)}\left(c \alpha+b\left(d+y_{0}(\alpha+b \lambda)\right)\right)\right), \\
y_{+}(t)= & \frac{1}{(\alpha+b \lambda)^{2}}\left(d(t \alpha(\alpha+b \lambda)-b \lambda)+\alpha\left(y_{0}(\alpha+b \lambda)-c \lambda(t \alpha+b t \lambda+1)\right.\right. \\
& \left.\left.+e^{t(\alpha+b \lambda)} \lambda\left(y_{0} \lambda b^{2}+\left(d+y_{0} \alpha\right) b+c \alpha\right)\right)\right),
\end{aligned}
$$


and the corresponding solution $\left(x_{-}(t), y_{-}(t)\right)$ of system (2) with the same initial condition is

$$
\begin{aligned}
x_{-}(t)= & \frac{1}{(a+b \lambda)^{2}}\left(-a\left(-b t \lambda c+c+b\left(d t+y_{0}\right)\right)-b\left(b t \lambda d+d+b \lambda\left(y_{0}-c t \lambda\right)\right)\right. \\
& \left.+e^{t(a+b \lambda)}\left(a\left(c+b y_{0}\right)+b\left(d+b y_{0} \lambda\right)\right)\right), \\
y_{-}(t)= & \frac{1}{(a+b \lambda)^{2}}\left(\left(d t-c \lambda t+y_{0}\right) a^{2}+\lambda\left(b\left(d t+y_{0}\right)-c(b t \lambda+1)\right) a-b d \lambda\right. \\
& \left.+e^{t(a+b \lambda)} \lambda\left(a\left(c+b y_{0}\right)+b\left(d+b y_{0} \lambda\right)\right)\right) .
\end{aligned}
$$

Let $t_{+}$be the time that the solution $\left(x_{+}(t), y_{+}(t)\right)$, starting at the point $\left(0, y_{0}\right)$ when $t=0$, enters in forward time in the half-plane $x>0$ and reaches by first time the straight line $x=0$, in case that such solution exists. Similarly, let $-t_{-}$be the time that the solution $\left(x_{-}(t), y_{-}(t)\right)$, starting at the point $\left(0, y_{0}\right)$ when $t=0$, enters in backward time in the half-plane $x<0$ and reaches by first time the straight line $x=0$, in case that such solution exists. Therefore, the piecewise linear differential system (1) and (2) has limit cycles if the system

$$
x_{+}\left(t_{+}\right)=0, \quad x_{-}\left(-t_{-}\right)=0, \quad y_{+}\left(t_{+}\right)-y\left(-t_{-}\right)=0,
$$

has isolated solutions. We have three equations and three unknowns $t_{+}, t_{-}$ and $y_{0}$.

From equation $x_{+}\left(t_{+}\right)=0$ we get that

$$
e^{t_{+}(\alpha+b \lambda)}=\frac{c \alpha+b\left((\alpha+b \lambda)\left(y_{0}-c t_{+} \lambda\right)+d\left(t_{+}(\alpha+b \lambda)+1\right)\right)}{c \alpha+b\left(d+y_{0}(\alpha+b \lambda)\right)},
$$

and from equation $x_{-}\left(-t_{-}\right)=0$ we obtain that

$$
e^{t_{-}(a+b \lambda)}=\frac{a\left(c+b y_{0}\right)+b\left(d+b y_{0} \lambda\right)}{\lambda\left(-d t_{-}+c \lambda t_{-}+y_{0}\right) b^{2}+d b+a\left(b t_{-} \lambda c+c+b\left(y_{0}-d t_{-}\right)\right)} .
$$

We note that if some of the denominators in the expressions of the previous both exponentials is zero, it follows easily that system (8) has no solutions.

Substituting these two exponentials in equation $y_{+}\left(t_{+}\right)-y\left(-t_{-}\right)=0$ we obtain that

$$
\left(t_{+}+t_{-}\right)(d-c \lambda)=0 .
$$

This is in contradiction with the facts that $t_{+}>0, t_{-}>0$ and $d-\lambda c \neq 0$, otherwise system (7) would have a straight line of equilibria. Then system (8) has no solutions, so the piecewise linear differential system has no limit cycles. This completes the proof of Theorem 1.

\section{Discontinuous piecenise differential system: Proof of}

THEOREM 2

Again we consider the planar piecewise differential systems formed by two linear differential systems separated by a straight line, such that both linear differential have no equilibria, neither real nor virtual, defined by (1) 
and (2). But now we assume that this piecewise differential systems are discontinuous, i.e. we do not consider the conditions (4).

Using the notation of the proof of Theorem 1 we have

$$
\operatorname{Div}_{1}=\alpha+\lambda \beta, \quad \operatorname{Div}_{2}=a+\mu b .
$$

Here, we must separate the proof of Theorem 2 in three cases.

Case 1: $\operatorname{Div}_{1}=\operatorname{Div}_{2}=0$. Then the solution $\left(x_{+}(t), y_{+}(t)\right)$ of system (1) with the initial condition $\left(x_{+}(0), y_{+}(0)\right)=\left(0, y_{0}\right)$ is

$$
\begin{aligned}
& x_{+}(t)=\frac{1}{2} t\left(2 y_{0} \beta+t \delta \beta+\gamma(2-t \beta \lambda)\right), \\
& y_{+}(t)=\frac{1}{2} \beta \lambda(\delta-\gamma \lambda) t^{2}+\delta t+y_{0} \beta \lambda t+y_{0},
\end{aligned}
$$

and the corresponding solution $\left(x_{-}(t), y_{-}(t)\right)$ of system (2) with the initial condition $\left(x_{-}(0), y_{-}(0)\right)=\left(0, y_{0}\right)$ is

$$
\begin{aligned}
& x_{-}(t)=\frac{1}{2} t\left(-b t \mu c+2 c+b d t+2 b y_{0}\right), \\
& y_{-}(t)=y_{0}+\frac{1}{2} t\left(d(b t \mu+2)+b \mu\left(2 y_{0}-c t \mu\right)\right) .
\end{aligned}
$$

We define the times $t_{+}$and $t_{-}$as in the proof of Theorem 1. So, now the discontinuous piecewise linear differential system (1) and (2) has limit cycles if the system

$$
x_{+}\left(t_{+}\right)=0, \quad x_{-}\left(-t_{-}\right)=0, \quad y_{+}\left(t_{+}\right)-y\left(-t_{-}\right)=0,
$$

with unknowns $t_{+}, t_{-}$and $y_{0}$ has isolated solutions.

From equation $x_{+}\left(t_{+}\right)=0$ we get that

$$
t_{+}=-\frac{2\left(y_{0} \beta+\gamma\right)}{\beta(\delta-\gamma \lambda)}
$$

and from equation $x_{-}\left(-t_{-}\right)=0$ we obtain that

$$
y_{0}=\frac{b(d-\mu c) t_{-}-2 c}{2 b} .
$$

We note that the denominator of $t_{+}$cannot be zero, otherwise $t_{+}$will be infinity, and we cannot have a periodic solution solution satisfying (10). Also the denominator of $y_{0}$ cannot be zero, otherwise $b=0$ and therefore $x_{-}(t)=c t$. So if $c \neq 0$ never holds $x_{-}\left(-t_{-}\right)=0$; and if $c=0$ we have that $x_{-}(t)=0$ for all $t$, so in this case we cannot have periodic solutions of the ones here consider with a piece in the half-space $x>0$ and another piece in the half-space $x<0$.

Substituting $t_{+}$and $y_{0}$ in equation $y_{+}\left(t_{+}\right)-y\left(-t_{-}\right)=0$ we have that

$$
\frac{c}{b}-\frac{\gamma}{\beta}=0 .
$$

Hence, if this last equality does not hold, then system (10) has no solutions and consequently the system has no periodic solutions. If $c / b-\gamma / \beta \neq 0$ then solving the second equation from (10) with respect to $t_{-}>0$ we get at 
most one solution, which substituted in (12) provides at most one periodic solution. So the theorem is proved in Case 1.

Case 2: $\operatorname{Div}_{1}=0$ and $\operatorname{Div}_{2} \neq 0$. Of course the case $\operatorname{Div}_{1} \neq 0$ and $\operatorname{Div}_{2}=0$ would follow in a similar way. Now the solution $\left(x_{+}(t), y_{+}(t)\right)$ of system (1) with the initial condition $\left(x_{+}(0), y_{+}(0)\right)=\left(0, y_{0}\right)$ is given in $(9)$, and the corresponding solution $\left(x_{-}(t), y_{-}(t)\right)$ of system $(2)$ with the same initial condition is

$$
\begin{aligned}
x_{-}(t)= & \frac{1}{(a+b \mu)^{2}}\left(b e^{t(a+b \mu)}\left(d+b y_{0} \mu\right)+a\left(-b\left(d t+y_{0}\right)\right.\right. \\
& \left.\left.+e^{t(a+b \mu)}\left(c+b y_{0}\right)+c(b t \mu-1)\right)-b\left(b t \mu d+d+b \mu\left(y_{0}-c t \mu\right)\right)\right), \\
y_{-}(t)= & \frac{1}{(a+b \mu)^{2}}\left(\left(d t-c \mu t+y_{0}\right) a^{2}+\mu\left(b\left(d t+y_{0}\right)\right.\right. \\
& \left.\left.+e^{t(a+b \mu)}\left(c+b y_{0}\right)-c(b t \mu+1)\right) a+b \mu\left(e^{t(a+b \mu)}\left(d+b y_{0} \mu\right)-d\right)\right) .
\end{aligned}
$$

Again we must find the isolated solutions $t_{+}, t_{-}$and $y_{0}$ of system (10).

From equation $x_{+}\left(t_{+}\right)=0$ we get that $t_{+}$is given in (11), and from equation $x_{-}\left(-t_{-}\right)=0$ we obtain that

$$
y_{0}=\frac{a c+b d-e^{(a+b \mu) t_{-}}\left(a c+b d+b(a+b \mu)(c \mu-d) t_{-}\right)}{b(a+b \mu)\left(e^{(a+b \mu) t_{-}}-1\right)} .
$$

Again we note that if some of the denominators in the expressions of $t_{+}$or $y_{0}$ is zero, it follows easily that system (8) has no solutions.

Substituting $t_{+}$and $y_{0}$ in equation $y_{+}\left(t_{+}\right)-y\left(-t_{-}\right)=0$ we have that

$$
\frac{2(a c+b d)}{b(a+b \mu)}-\frac{2 \gamma}{\beta}+(c \mu-d) t_{-} \operatorname{coth}\left(\frac{1}{2}(a+b \mu) t_{-}\right)=0
$$

Since the function $z \operatorname{coth}(z)$ for $z>0$ is strictly increasing, and at $z=0$ takes the zero value, it follows that equation (14) has at most one solution for $z=\frac{1}{2}(a+b \mu) t_{-}$(i.e for $t_{-}$), and consequently a unique solution for $y_{0}$ and $t_{+}$from (13) and (11), respectively. Obtaining in this way at most one limit cycle for the discontinuous piecewise linear differential system (1) and (2), hence Theorem 2 is proved under the assumptions of Case 2.

We note that the discontinuous piecewise linear differential system which provides the limit cycle of Figure 2 is a particular system of the ones studied in this Case 2. 
Case 3: $\operatorname{Div}_{1} \operatorname{Div}_{2} \neq 0$. Now the solution $\left(x_{+}(t), y_{+}(t)\right)$ of system (1) with the initial condition $\left(x_{+}(0), y_{+}(0)\right)=\left(0, y_{0}\right)$ is

$$
\begin{aligned}
x_{+}(t)= & \frac{1}{(\alpha+\beta \lambda)^{2}}\left(t \gamma \lambda^{2} \beta^{2}-t \delta \lambda \beta^{2}-t \alpha \delta \beta-\delta \beta+t \alpha \gamma \lambda \beta\right. \\
& \left.+\left(e^{t(\alpha+\beta \lambda)}-1\right) y_{0}(\alpha+\beta \lambda) \beta-\alpha \gamma+e^{t(\alpha+\beta \lambda)}(\alpha \gamma+\beta \delta)\right), \\
y_{+}(t)= & \frac{1}{(\alpha+\beta \lambda)^{2}}\left(\left(-1+e^{t(\alpha+\beta \lambda)}\right)(\alpha \gamma+\beta \delta) \lambda\right. \\
& \left.+y_{0}(\alpha+\beta \lambda)\left(\alpha+e^{t(\alpha+\beta \lambda)} \beta \lambda\right)+t \alpha(\alpha+\beta \lambda)(\delta-\gamma \lambda)\right),
\end{aligned}
$$

and the corresponding solution $\left(x_{-}(t), y_{-}(t)\right)$ of system $(2)$ with the initial condition $\left(x_{-}(0), y_{-}(0)\right)=\left(0, y_{0}\right)$ is the same than in the previous Case 2.

Defining $t_{+}$and $t_{-}$as before the piecewise linear differential system (1) and (2) has limit cycles if system (8) has isolated solutions for the unknowns $t_{+}, t_{-}$and $y_{0}$.

We change the parameters $\alpha$ and $a$ by $u^{2}$ and $v^{2}$ defined as $\alpha=u^{2}-\beta \lambda$ and $a=-v^{2}-b \mu$, respectively. From equation $x_{+}\left(t_{+}\right)=0$ we get that

$$
e^{u^{2} t_{+}}=\frac{\beta(\delta-\gamma \lambda) u^{2} t_{+}}{\left(y_{0} \beta+\gamma\right) u^{2}+\beta(\delta-\gamma \lambda)}+1,
$$

and from equation $x_{-}\left(-t_{-}\right)=0$ we obtain that

$$
e^{v^{2} t_{-}}=\frac{b(c \mu-d) v^{2} t_{-}}{c v^{2}+b\left(y_{0} v^{2}-d+c \mu\right)}+1 .
$$

We note that if some of the denominators in the expressions of the previous both exponentials is zero, the present study can be done in a similar and easier way.

Substituting these two exponentials in equation $y_{+}\left(t_{+}\right)-y\left(-t_{-}\right)=0$ we have that

$$
u^{4} v^{4}\left((d-c \mu) t_{-}+(\delta-\gamma \lambda) t_{+}\right)=0 .
$$

From this last equation we obtain that

$$
t_{+}=\frac{(d-c \mu) t_{-}}{\gamma \lambda-\delta}>0 .
$$

Isolating $y_{0}$ from equation $x_{-}\left(-t_{-}\right)=0$, substituting $t_{+}$and $y_{0}$ in equation $x_{+}\left(t_{+}\right)=0$, and changing the variable $t_{-}$by the variable $z$ through

$$
t_{-}=\frac{z(\gamma \lambda-\delta)}{u^{2}(d-c \mu)}>0,
$$

we obtain the equation

$$
c_{0} f_{0}+c_{1} f_{1}=0,
$$

where

$$
\begin{aligned}
& c_{0}=u^{2}\left(b\left(\gamma v^{2}+d \beta-c \beta \mu\right)-c v^{2} \beta\right), \\
& c_{1}=b v^{2} \beta(\delta-\gamma \lambda),
\end{aligned}
$$


and

where

$$
\begin{aligned}
& f_{0}(z)=\left(e^{z}-1\right)\left(e^{k}-1\right), \\
& f_{1}(z)=1+e^{k}\left(-z+e^{z}-1\right)+e^{z}(z-1),
\end{aligned}
$$

$$
k=\frac{v^{2}(\gamma \lambda-\delta)}{u^{2}(d-c \mu)}>0 .
$$

We note that in the particular case that $k=1$ equation (17) becomes

$$
c_{0} f_{0}+c_{1} f_{1}=\left(c_{0}+c_{1}\right)\left(e^{z}-1\right)^{2}=0,
$$

which has no solutions for $z>0$.

Now we shall prove that the functions $f_{0}$ and $f_{1}$ form an Extended Complete Chebyshev system if $k \neq 1$. So, if $k \neq 1$ the equation (17) can have at most one zero, and this can be reached. See for more detalls on Chebyschev system the appendix. In short, if the equation (17) as at most one solution $t_{-}$, from it we get a unique value for $y_{0}$ and $t_{+}$, and consequently at most a unique limit cycle for the discontinuous piecewise linear differential system (1) and (2) under the assumptions of Case 3. This completes the proof of Theorem 2.

Only remains to show that the functions $f_{0}$ and $f_{1}$ form an Extended Complete Chebyshev system if $k \neq 1$. Indeed, from equation (15) and since $t_{+}$and $t_{-}$are positive (by definition), it follows that $(d-c \mu)(\gamma \lambda-\delta)>0$. Therefore, from (16) we get that $z>0$ and $k>0$. Now we change the variable $z$ by the new variable $t$ through $z=\log t$ with $t>1$. So, in function of the new variable $t$ we have

$$
\begin{aligned}
& f_{0}(t)=(t-1)\left(t^{k}-1\right)>0, \\
& f_{1}(t)=(t-1)\left(t^{k}-1\right)+\left(t-t^{k}\right) \log t .
\end{aligned}
$$

Hence, from Theorem 5 of the appendix, in order to see that the functions $f_{0}$ and $f_{1}$ form an Extended Complete Chebyshev system for $t>1$ we only need to show that the Wronkskian

$$
\begin{aligned}
W(t) & =\left|\begin{array}{ll}
f_{0}(t) & f_{1}(t) \\
f_{0}^{\prime}(t) & f_{1}^{\prime}(t)
\end{array}\right| \\
& =(t-1)\left(t^{k}-1\right)\left(t-t^{k}\right)+\left(t\left(t^{k}-1\right)^{2}-k(t-1)^{2} t^{k}\right) \log t,
\end{aligned}
$$

does not vanish for $k>0$ and $k \neq 1$. Since we have that $W(t)<0$ if $k \in(0,1), W(t)=0$ if $k=1$, and $W(t)>0$ if $k>1$ (see also Figures 3 and 4 , the functions $f_{0}$ and $f_{1}$ form an Extended Complete Chebyshev system if $k \neq 1$.

\section{Appendix Extended Complete Chebyshev system}

The functions $\left(f_{0}, \ldots, f_{n}\right)$ defined on an interval $I$ form an Extended Chebyshev system if and only if any non-zero linear combination of these functions has at most $n$ zeros in $I$ taking into account their multiplicities and this number is reached. 


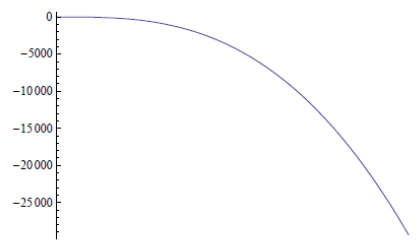

FiguRE 3. The graphic of $W(t)$ when $k \in(0,1)$.

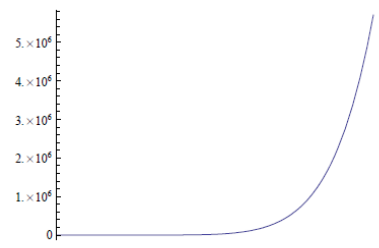

FiguRE 4. The graphic of $W(t)$ when $k>1$.

The functions $\left(f_{0}, \ldots, f_{n}\right)$ form an Extended Complete Chebyshev system if and only if for any $k \in\{0,1, \ldots, n\},\left(f_{0}, \ldots, f_{k}\right)$ form an Extended Chebyshev system.

Theorem 5. Let $f_{0}, \ldots, f_{n}$ be analytic functions defined on an open interval $I \subset \mathbb{R}$. Then $\left(f_{0}, \ldots, f_{n}\right)$ is an Extended Complete Chebyshev system on $I$ if and only if for each $k \in\{0,1, \ldots, n\}$ and all $y \in I$ the Wronskian

is different from zero.

$$
W\left(f_{0}, \ldots, f_{k}\right)(y)=\left|\begin{array}{cccc}
f_{0}(y) & f_{1}(y) & \cdots & f_{k}(y) \\
f_{0}^{\prime}(y) & f_{1}^{\prime}(y) & \cdots & f_{k}^{\prime}(y) \\
\vdots & \vdots & \ddots & \vdots \\
f_{0}^{(k)}(y) & f_{1}^{(k)}(y) & \cdots & f_{k}^{(k)}(y)
\end{array}\right|
$$

For a proof of Theorem 5 see [16].

\section{ACKNOWLEDGMENTS}

We thank to the reviewers their good comments which help us to improve the presentation of this paper.

The first author is partially supported by a MINECO/FEDER grant MTM2013-40998-P, an AGAUR grant number 2014SGR568, the grants FP7PEOPLE-2012-IRSES 318999 and 316338, and a CAPES grant 88881. 030454/ 2013-01 do Programa CSF-PVE. The second author is partially supported by FAPESP under grant number 2012/18780-0.

\section{REFERENCES}

[1] A. Andronov, A. Vitt And S. Khaikin, Theory of Oscillations, Pergamon Press, Oxford, 1966.

[2] J. C. Artés, J. Llibre, J. C. Medrado and M. A. Teixeira, Piecewise linear differential systems with two real saddles, Mathematics and Computers in Simulation 95 (2013), 13-22.

[3] D.C. Braga And L. F. Mello, Limit cycles in a family of discontinuous piecewise linear differential systems with two zones in the plane Nonlinear Dynam. 73 (2013), 1283-1288.

[4] C. Buzzi, C. Pessoa And J. Torregrosa, Piecewise linear perturbations of a linear center, Discrete Contin. Dyn. Syst. 33 (2013), 3915-3936. 
[5] M. di Bernardo, C. J. Budd, A. R. Champneys and P. Kowalczyk, PiecewiseSmooth Dynamical Systems: Theory and Applications, Appl. Math. Sci. Series 163, Springer-Verlag, London, 2008.

[6] R.D. EuzÉBio AND J. Llibre, On the number of limit cycles in discontinuous piecewise linear differential systems with two pieces separated by a straight line, J. Math. Anal. Appl. 424 (2015), 475-486.

[7] E. Freire, E. Ponce, F. Rodrigo And F. Torres, Bifurcation sets of continuous piecewise linear systems with two zones, Int. J. Bifurcation and Chaos 8 (1998), 20732097.

[8] E. Freire, E. Ponce And F. Torres, Canonical Discontinuous Planar Piecewise Linear Systems, SIAM J. Applied Dynamical Systems 11 (2012), 181-211.

[9] E. Freire, E. Ponce And F. Torres, The discontinuous matching of two planar linear foci can have three nested crossing limit cycles, Publ. Mat. Vol. extra (2014), $221-253$.

[10] E. Freire, E. Ponce And F. Torres, A general mechanism to generate three limit cycles in planar Filippov systems with two zones, Nonlinear Dynamics 78 (2014), 251-263.

[11] F. Giannakopoulos And K. Pliete, Planar systems of piecewise linear differential equations with a line of discontinuity, Nonlinearity 14 (2001), 1611-1632.

[12] M. HAN AND W. ZHANG, On Hopf bifurcation in non-smooth planar systems, J. of Differential Equations 248 (2010), 2399-2416.

[13] S. M. HuAn AND X. S. YAnG, On the number of limit cycles in general planar piecewise linear systems, Discrete and Continuous Dynamical Systems-A 32 (2012), $2147-2164$.

[14] S. M. HuAn AND X. S. YANG, Existence of limit cycles in general planar piecewise linear systems of saddle-saddle dynamics, Nonlinear Anal. 92 (2013), 82-95.

[15] S. M. HuAn AND X. S. YANG, On the number of limit cycles in general planar piecewise linear systems of node-node types, J. Math. Anal. Appl. 411 (2014), 340353.

[16] S.J. Karlin and W.J. Studden, T-Systems: With Applications in Analysis and Statistics, Pure Appl. Math., Interscience Publishers, New York, London, Sidney, 1966.

[17] R. E. Leine and D. H. van Campen, Discontinuous bifurcations of periodic solutions, Mathematical and Computing Modelling 36 (2002), 259-273.

[18] J. Llibre, D.D. Novaes and M.A. Teixeira, On the birth of limit cycles for nonsmooth dynamical systems, Bull. Sci. Math. 139 (2015) 229-244.

[19] J. Llibre, D.D. Novaes AND M.A. TeixeIRA, Limit cycles bifurcating from the periodic orbits of a discontinuous piecewise linear differential center with two zones, Int. J. Bifurc. Chaos, 25 (2015), 1550144-pp 11.

[20] J. Llibre, D.D. Novaes AND M.A. TeIXeIRA, Maximum number of limit cycles for certain piecewise linear dynamical systems, Nonlinear Dynamics 82 (2015), 11591175.

[21] J. Llibre, M. Ordóñez And E. Ponce, On the existence and uniqueness of limit cycles in planar continuous piecewise linear systems without symmetry, Nonlinear Analysis: Real World Applications 19 (2012), 325-335.

[22] J. Llibre AND E. Ponce, Three nested limit cycles in discontinuous piecewise linear differential systems with two zones, Dynamics of Continuous, Discrete \& Impulsive Systems, Series B 19 (2012), 325-335.

[23] J. Llibre And J. Sotomayor, Phase portraits of planar control systems, Nonlinear Analysis, Theory, Methods and Applications 27 (1996), 1177-1197.

[24] J. Llibre, M.A. Teixeira And J. Torregrosa, Lower bounds for the maximum number of limit cycles of discontinuous piecewise linear differential systems with a straight line of separation, Int. J. Bifurcation and Chaos 23 (2013), 1350066-10 pp. 
[25] R. Lum, L. O. ChuA, Global properties of continuous piecewise-linear vector fields. Part I: Simplest case in $R^{2}$, Memorandum UCB/ERL M90/22, University of California at Berkeley, 1990.

[26] O. Makarenkov and J.S.W. Lamb, Dynamics and bifurcations of nonsmooth systems: A survey, Physica D 241 (2012), 1826-1844.

[27] J.E. Marsden and A. Tromba, Vector Calculus, 5th edition, W. H. Freeman, New York, 2003.

[28] D.J.W. Simpson, Bifurcations in Piecewise-Smooth Continuous Systems, World Scientific Series on Nonlinear Science A, vol 69, World Scientific, Singapore, 2010.

${ }^{1}$ Departament de Matemàtiques, Universitat Autònoma de Barcelona, 08193 Bellaterra, Barcelona, Catalonia, Spain

2 Departamento de Matemática, Universidade Estadual de Campinas, Caixa Postal 6065, 13083-970, Campinas, SP, Brazil

E-mail address: jllibre@mat.uab.cat

E-mail address: teixeira@ime.unicamp.br 